\title{
REINFORCEMENT LEARNING FOR ROBOTIC Grasping AND MANipulation: A REVIEW
}

Asia Pac. j. energy environ.

\section{Praveen Kumar Donepudi}

Enterprise Architect, Information Technology, UST-Global, Inc., Ohio, USA

*Email for Correspondence: praveen.donepudi@ust-global.com

Abstract

A century of robots is the 21st century. The robots have long been able to cross the divide between the virtual universe and the real world. Robotics, as the most successful contender in the upcoming great technological revolution, will play an ever more important role in society because of the impact it has in every field of life, including medicine, healthcare, architecture, manufacturing and food supplies, logistics and transport. This document introduces a modern approach to the grasp of robots, which draws grasp techniques from the human demonstration and combines these strategies into a grasp-planning framework, in order to produce a viable insight into the objective geometry and manipulation tasks of the object. Our study findings show that grasping strategies of the form of grasp and thumbs positioning are not only necessary for human grasp but also significant restrictions on posture and wrist posture which greatly reduce both the robot hand's workplace and the search space for grasp planning. In the simulation and with a true robotic system this method has been extensively tested for several everyday living representative objects. In the experiment with varying degrees of perceiving in certainties, we have demonstrated the power of our method.

Key words

Grasp planning, Machine learning, Reinforcement learning

This article is is licensed under a Creative Commons Attribution-NonCommercial 4.0 International License.

Attribution-Non Commercial (CC BY-NC) license lets others remix, tweak, and build upon work non-commercially, and although the new works must also acknowledge \& be non-commercial.

\section{INTRODUCTION}

For the selection of tasks in warehouses or industry, robotic grasping is essential. But the challenge continues to be met with robotic gripping under unstructured conditions for changes in the atmosphere and lighting, vast quantities of objects with diverse properties, dynamic surroundings, and occlusion between objects and so on. This all adds to immense confusion about how to achieve popularity. In this area, early efforts are made to fit 3D model to objects or to carry out 3D analytical considerations to predict the region of capture. However, for 3D template mattering, a large search library and a geometry-based approach to a wide variety of density and mass distribution assumptions, which can be relevant to robotic comprehend, typically require time and effort. As a result, there have been more and more approaches to machine learning with fantastic breakthroughs (LeCun et al., 2015). In an unfamiliar scene, robotic grasping includes perceiving the setting, motion preparation and some robot control problems. In this post, we focus primarily on the issue of perception and partly on robot control with reverse filmmaking. Perception is important to communicate with environments for robotic grippers. In the investigation of robots the motivation of visual detection is to define right locations for objects. The right grip poses for new objects are a simple job for human beings. But a visual task as grasp-detection is a challenging topic for robotics in recent years for unknown objects. In general, it is important to rapidly and reliably detect grasping of unfamiliar objects on a tabletop scene. If an item stack exists, it is necessary to figure out where it is and to isolate it, in order to find a structure for each piece. The easiest way to understand each item is to find the right location, then reach it and close the pin. The technique will guarantee the success rate when we decide to grasp objects in a common configuration if we grasp the same object (Daniilidis et al., 1999). 


\section{LITERATURE REVIEW}

The topic of robot grabbing in literature has been extensively discussed and is an active area of study. One camp tackles the grasping by empirical process (Bicchi A, 1995) to allow use of force and shape closures to maintain a secure grip. Such methods presume that touch points on the object are possible and depend on well-defined 3D models of the object, which in real-time situations are usually not available. A further line of study is focused on the database (Bohg et al., 2014), which also takes into account the existence of full 3D object models and uses a precomputed grasp dataset. Some programs use grab data and usually need a manually labelled grabbing dataset to learn grab detection models (Saxena et al., 2008). Approaches recently use profound learning methods, which require massive training datasets and which are not typically suited for applications in real time. (Lenz et al., 2015).

Similar to our work, other methods are an entity with basic types. The primitive types for grasp pickup need established object models as well. Various primitives are assigned by the writers (Yamanobe \& Nagata, 2010). The authors create 3D data points to constellate primitive boxes for object grasping by pieces. In addition, approaches depend on simulation tools that does not always fit well with the physical output of the robot grasp

There are approaches which use cloud-point data to detect grasp. Our job involves neither learning nor meshing nor running on cloud input data directly. The authors (Donepudi, 2018) intend to capture household objects with an assistive robot that does not rely on object modelling and senses a single overhead grip in objects' clouds. A top view of the target (caught with a laser scanner) is the solution and the user will show which object using a laser pointer. In relation to encounters with humans, there exist studies of legible and predictable goal-oriented gestures (motions that express their purpose to the human observer) by the robot (Dragan et al., 2013). The robot must also understand precisely the purpose of humans It is also necessary.

Our strategy aims to accomplish these two goals by producing applicant understandings close to what users produce themselves and choosing candidate understandings based on the assumed user's purpose. We also have a peculiar strategy in that it creates several candidate grip poses. We assume that the identification of a particular target will be unsatisfactory in collaborator human-robot tales, and that the choice of the human position will differ as the world varies (Balasubramanian et al., 2010).

\section{Robotic Grasping by LetTINg Robots LEARN FOR ThEMSELVES}

Robots can typically be trained to carry out a specific work, although they may be drafted with the aid of machine learning to write the instructions themselves. Vicarious AI developers, a California-based robotic start-up, have developed a computing interface linked to the cameras system and a robotic gripper called a Visual Cognitive Machine (VCC). Human beings are good at inferring and then applying the ideas that are expressed into a pair of images (Donepudi, 2019). The principles inferred by humans are high enough to easily be used in circumstances which look somewhat different, so that the language-independent instructions are used by IKEA and LEGO. Don't lift your expectations, though, these robots cannot even bring you together with the flat-pack table or the chair. But it can do very simple work, such as reverse and forward transfer a block. It functions that way. Next, an input and output picture would be obtained by the system.

The input image is a jumble of cultured items of all kinds and sizes, while an ordered set of objects is the output image. For example, several red blocks can be the input image, and all the red blocks ordered for a circle are the output image. Before and after, think about it a little like a snapshot. Based on the 'front' to the "after" image, the VCC determines the commands that the robot wants to execute to coordinate the object placed before it. The computer is designed to understand what action is required by supervised learning.

The diagram of all the different components operating the robot is on the left. The visible hierarchy views and categorizes objects in front of the camera according to shape and color. When the fixing controller concentrates on objects before the manual controller operates the robot's arms to move objects, it sets which objects to concentrate.

Just 24 commands on the right side are available for the VCC controller, and the robot does not have to work with too many samples. The physical objects that are placed before the robot must not be the same as abstract objects used in the input and output images. In order to see how robots could conform to a more natural world, researchers created 546 separate tasks or ideas from 4 lines of instructions to 23 with varying numbers and sizes of objects and background color and textures. Six were tested on two separate robots from 546 ideas. One was the Rethink Robotics platform from Baxter and the other was the Universal Robots UR5 robo "We have spoken independently on both of those ideas," said George. "We then wrote small programs to produce different images to match each definition. 
A

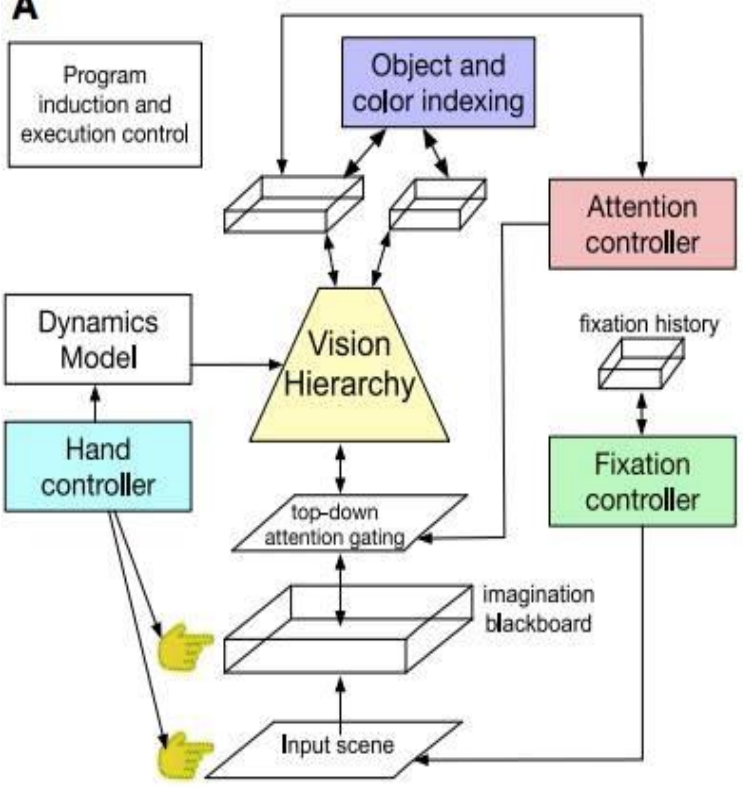

B

\section{Instruction set}

\begin{tabular}{|c|c|}
\hline Vision Hierarchy. & Hand conrol \\
\hline top_down_attend & $\overline{\text { grab_object }}$ \\
\hline fill_color () & release_object \\
\hline imagine_object() & move_hand_to_fixation \\
\hline Attention control & move_hand_left \\
\hline set_shape_attn() & move_hand_right \\
\hline set_color_attn() & move_hand_up \\
\hline reset_attn & move_hand_down \\
\hline Fixation control & move_hand_to_attended_object \\
\hline $\begin{array}{l}\text { fixate_attended_object } \\
\text { fixate_previous }\end{array}$ & Object indexing \\
\hline fixate_next & loop_start \\
\hline fixate_pointer & loop_end \\
\hline fixate_location() & next_object \\
\hline
\end{tabular}

Figure 1: A: graph describing the robot's components. B: The list of commands the VCC can use

In contrast with the paper statements of Baxter, UR5 was better able to accomplish the tasks and could achieve more than $90 \%$ of the six concepts tested. Hardware malfunctions were constant errors, such as objects falling from the gripper. The Vicarious' Baxter robot was older and with time the camera was blurring and its movements less correct. "One of the aims of artificial intelligence and robot science is to accomplish robotics to execute tasks without specific programming". Other approaches have been implemented, such as mimic learning, where agents learn by copied presentations. However, these bots are less able to react to conditions outside the context of the demonstrations. Here, no clear demonstrations are available, which the scientists call "zero-shot learning." The organization must study and encode abstract ideas and then transfer the paradigm into situations in the real world. It sounds pretty cool, but the assignments are still very rudimentary and early in the day. It will be a while before factories excel in AI robots like these. Vicarious has said that it would test certain improvements in the idea in minimal circumstances" (Quach, 2019).

\section{Methodology}

We proposed a method to learn robotic grasping coordination with the eyes, using deep learning to create a grasping network and an ongoing servo system to guide a robotic manipulator continuously across the network. Our first experimental series consisted of training the device with nearly 800,000 grab attempts by 14 separate camera-variant robotic manipulators. These tests demonstrate that our approach can work with minor differences in hardware, camera positioning and wear and tear when the camera is not calibrated.

.In various types of industry, robotic grasping is widely used by following two different methods of operation; one is guided instructed operation and second is self-learning mechanism. In guided instructed operation, a specific set of instructions have been inserted in a microcontroller chip of a robot that produce well guided series of actions, regardless of the outside world. This type of method is very useful where the environment doesn't change and same action requires every single time (Maleque et al., 2010).

Whenever, we need to handle different tasks without any human interaction and let robot decide how to perform their action, we use a Self-learning mechanism. Whenever human involves in object handling behavior, the interaction mainly involves a feedback loop between experience and action. As they manipulate tasks, like extracting any single thing from a targeted bin, can be executed by continuous sensing such as touch and feel (Donepudi, 2018). It gives them a path to do that task more rapidly afterwards. In contrast, robotic grasping this manipulation mainly relies more on advance analysis of extracted large data set from a continuous experiments sequences, with relatively simple feedback, to ensure stability during action. For this continues feedback system, we used a real time imaging camera to track and analyze robotic grasp moment. Through this mechanism we developed a very large data sets consisting of different types of information necessary to perform our actions like coordinate axis, speed, angle, dimension and shapes (Ioffe and Szegedy, 2015). 


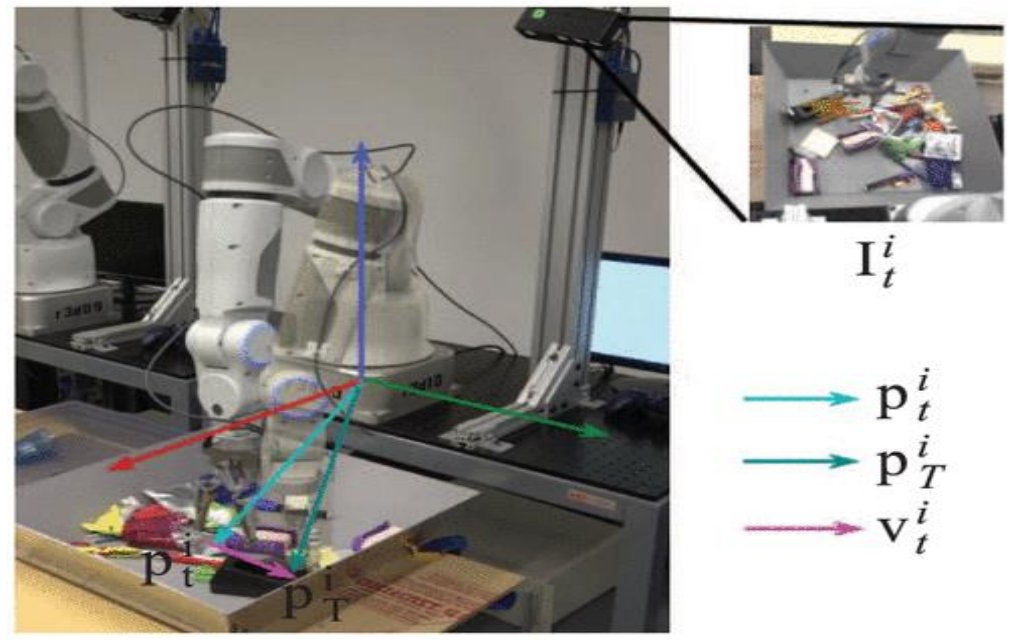

Figure 2: Diagram of the grasp sample setup

These types of information are gathered by different types of sensors in which mainly is a real time imaging camera. Mainly in this type of operation we convolute Robotic servo motor actions with our sensing camera input. Actions are totally dependent on our receiving data sets from sensors (Donepudi, 2017). Through these repeated exercises, we developed a self-learning environment for our robots and these data set analyses can be used on any outside world regardless of the robotic shape and camera resolution properties. Robot can adjust its grasping hand position and force accordingly to the targeted object shape and dimension by following its real time feedback system.

\section{Applications of Robotic Grasping in Different FieldS}

Robots can be categorized by their surroundings. The most common difference is between fixed and mobile robots. These two robots have very different operating conditions, and thus very different capacities are required. Fixed robots are primarily commercial robotic manipulators operating in well-defined robotic environments. Industrial robotics execute basic routine tasks in car manufacturing plants such as soldering or painting parts. Robot manipulators are commonly used in less regulated settings, for example high-precession surgery, as sensors and equipment for human robot contact are enhanced.

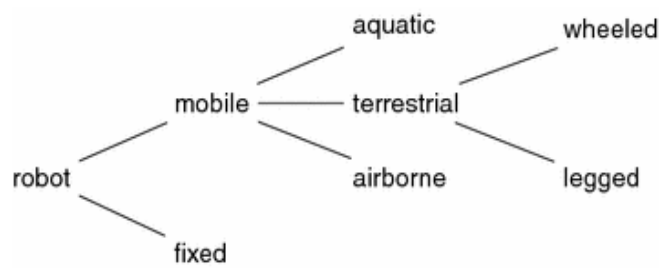

Figure 3: Classification of robots by environment and mechaism of interaction

In comparison, mobile robots are supposed to travel about and execute tasks that are not expressly built for robots in large, undefined and unknown settings. You must fix conditions which are not exactly understood in advance and which evolve over time. Unpredictable beings such as humans and animals may involve such environments. For example, robotic vacuum cleaners and self-drive cars are mobile robots.

In comparison, mobile robots are supposed to travel about and execute tasks that are not expressly built for robots in large, undefined and unknown settings. You must fix conditions which are not exactly understood in advance and which evolve over time. Unpredictable beings such as humans and animals may involve such environments. Robotic vacuum cleaners, for example, and handheld robots are self-moving vehicles.

The functions of fixed robots and mobile robots are not directly different-humans can interact with industrial robots and mobile robots can be forced to detect motion-but both classes are drastically different. However, looking at them is quick. In order to calculate their role in relation to their internal condition, fixed robots are particularly attached to their stability on the floor and the environmental consciousness of mobile robots has been determined by them.

Mobile robots require three essential conditions, since they differ with the method of movement: aquatic (underwater), terrestrial (car) and aerial robots (drones). Again, the concept of amphibious robots on and off the ground for 
example is not comprehensive. Robots can be additionally sub classed in all three environments; land-based robots with legs or limbs, as well as air spheres, which in turn are known as fixed-wing, helicopters, can be lighter than or heavier than airplanes. Their robots can also be sub classified.

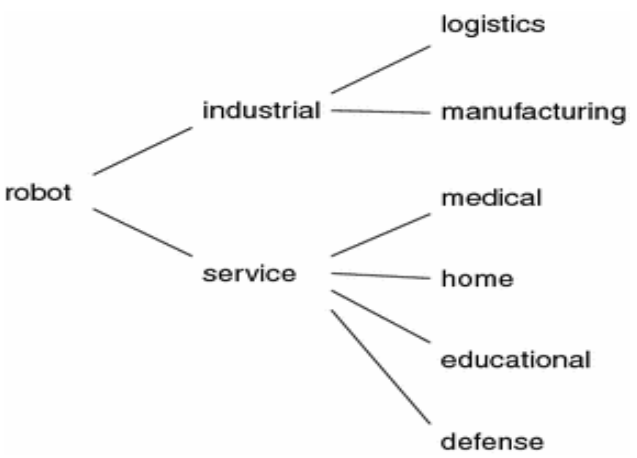

Figure 4: Classification of robots by application field

\section{INDUSTRIAL ROBOTS}

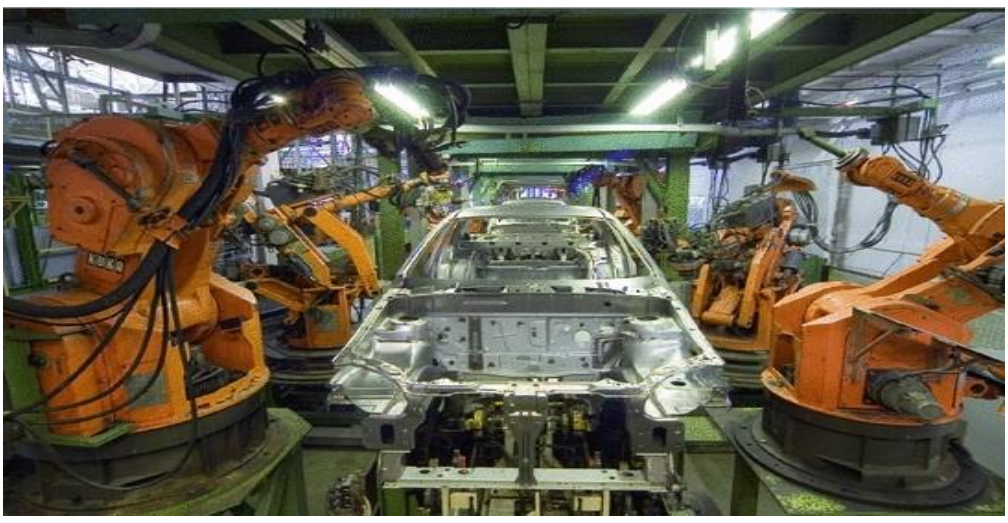

Figure 5: Robots on an assembly line in a car factory

Industrial robots were the first, replacing repetitive workers with employees. In a well-defined world, without the involvement of humans, the robot can perform tasks in a given order, using objects which are positioned exactly before it (Asadullah et al., 2019).

It may be argued that this is automatic rather than robotic. Today, though, automatons also rely on sensors so that they can be used as robots. Their architecture is therefore simplified by operating in a custom environment that people cannot enter whilst the robot works.

Today, however, more functionality is required for robots, such as the ability to manipulate objects in different directions or to classify different objects in the proper order. It can be required from and to the warehouses by the robot. The world is limited more or less and tailored to the robot, but the fundamental property persists.

There is a need for greater stability as industrial robots communicate with humans, and this entails strict safety criteria for both autonomous and mobile robots. In specific, the robot 's speed must be decreased and the mechanical architecture should ensure that the user does not endanger moving parts. The benefit of people working with robotics is that anyone can do the best: robots do tedious or risky works, while people take tougher steps to identify the robot 's overall tasks as mistakes and improvement choices are easily found.

\section{Autonomous MobiLe RoBots}

Many mobile robots are remotely tracked and conduct activities such as pipe analysis, air images and bomb disposal using a system controller. This robots are not autonomous; they are able to reach unsafe, distant, or inaccessible from their sensors remotely. Some can be semi-autonomous and automatically execute subscriptions. The drone autopilot stabilizes the flight and the man selects the route to the flight. An inside-a-pipe robot can monitor its movements while human beings are searching for defects to be remedied. Completely autonomous mobile robots do not depend on a boss, but rather take their own decisions and perform tasks like bringing 
material on dangerous land and at a continuously shifting landscape (people walking and vehicles walking on streets). (Their walls and doors within the houses, intersections on the streets).

The first mobile robots have been developed for basic environment applications, such as robots with washed pools or robotic paddling machines. Robot aspirator are currently very popular since it has been demonstrated that it is feasible to create reasonably priced robots that can man oeuvre indoors with obstacles.

Many mobile autonomous robots assist professionals in organized settings such as warehouses. A robot for weeding fields is an fascinating example. This ecosystem is partly organized, but sophisticated sensing is needed to detect and eliminate weeds. Also in very organized industries, robots share the world with people and must therefore be highly reliable in sensing.

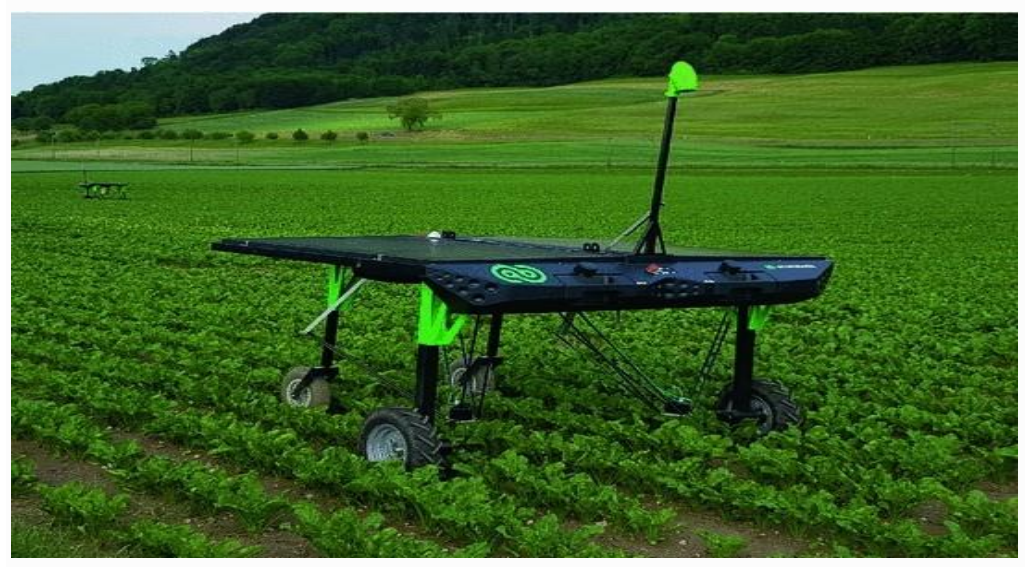

Figure 6: Autonomous mobile robot weeding a field

Perhaps the most common self-driving vehicle today is the autonomous mobile robot. Due to the very complicated motorized transport environment and the stringent safety criteria, they are very difficult to build.

Space is an even tougher and more risky setting. Semi-autonomous handheld robots are the Sojourner and Curiosity Mars rovers. Three months in 1997, the Sojourner was active. Since its arrival in Mars 2012, Curiosity has been alive! Where on Earth a human pilot manages missions (driving routes and research experiments), the rovers have the ability to avoid autonomous hazards. Many of the research and development currently under way in robotics focuses on increasing the freedom of robots through the enhancement of sensors and the intelligent control. Better sensors can sense specifics in more complicated situations, but the robot control must be highly versatile and adaptable in order to manage these situations. Vision, specifically, is a very active research area because the cameras are low costs and very rich knowledge they can collect. Efforts are being made to adaptive frameworks in order to benefit from individuals or to respond to new circumstances. The relationship between human beings and robots is another active area of study. This encompasses both senses and awareness, but the psychology and sociology of contact must still be taken into account.

\section{HUMANOID ROBOTS}

Robots like to portray scientist fiction and mass media in a humanoid shape. We all remember the robotic characters R2-D2 and 3-CPO in the Star Wars films, but the idea goes even deeper. In the 18th century Swiss watchmakers, Pierre and Henri-Louis, designed humanoid automatons in order to demonstrate their mechanical abilities and market their watches. They built the humanoid automatons of Jean-Frédéric Leschot, a group of them. For related purposes, many firms now manufacture humanoid robots. Humanoid robots are a compact autonomous robot with an incredibly complex mechanical configuration for arms movement and leg locomotion. Humanoid robots are used for studies into walking dynamics and the relationship of humans and computers. For operation and repair in a housing or space station, humanoid robots have been suggested. They are built to treat elderly people who could feel concerned in the presence of an inhuman computer. In the other side, robots quite like people will produce repulsion, a phenomena known as the uncanny valley.

The architecture and control of humanoid robots is very complicated. They are costly to create with many joints that can pass in a number of ways. Most systems favor robotics using wheels or tracks because they are faster, cheaper and durable (Ben-Ari \& Mondada, 1970). 


\section{HEALTHCARE INDUSTRY}

In the world of healthcare, new technologies and technical advancements are being increasingly developed. In the ongoing growth of this industry, robots have been an important player. Intuitive da Vinci robots for instance are operational robots that are used by physicians to conduct minimally disruptive prostatectomies and are considered the norm of treatment. They will also assist a surgeon in hysterectomies, chest surgery and other operations. Another less invasive robotic invention which has improved the health sector is the robot iRobot, a remote robot for out-patients. And from tremendous distance, this robot allows doctors to have a more personalized experience. During the 2020 coronavirus pandemic the need for this form of telemedicine grew.

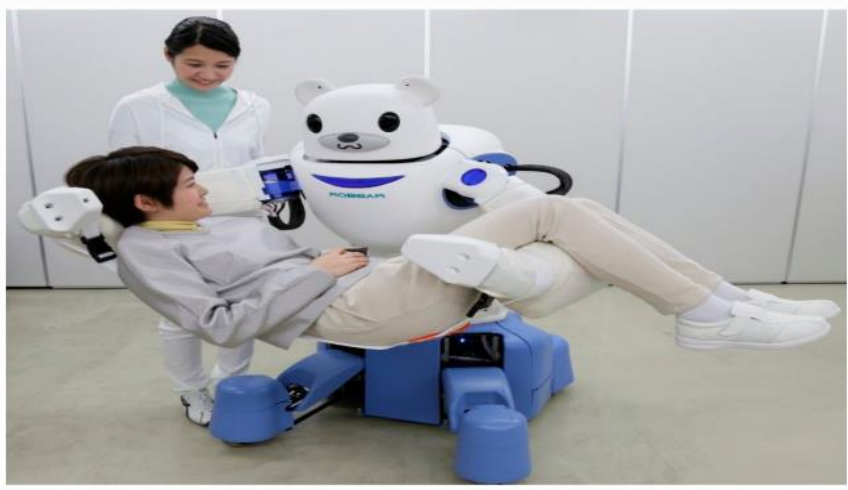

(a)

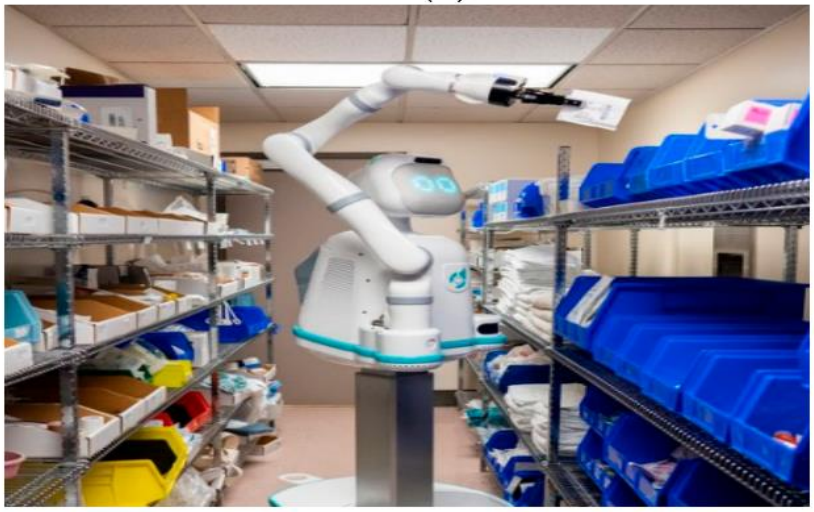

(c)

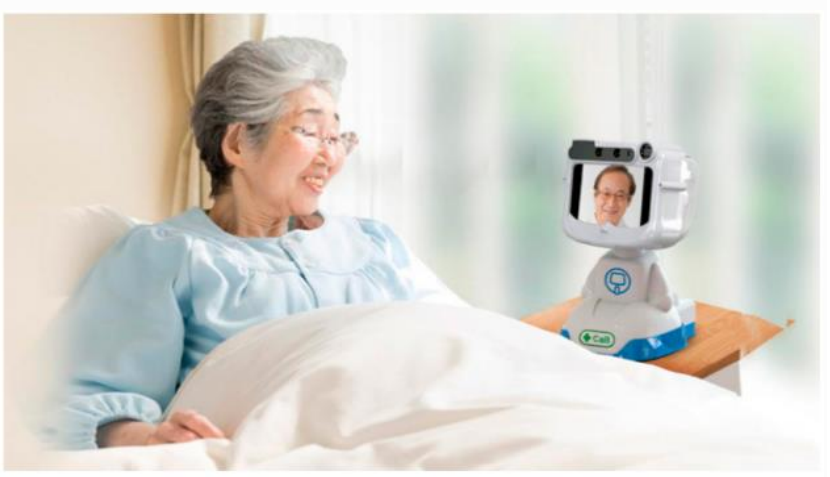

(b)

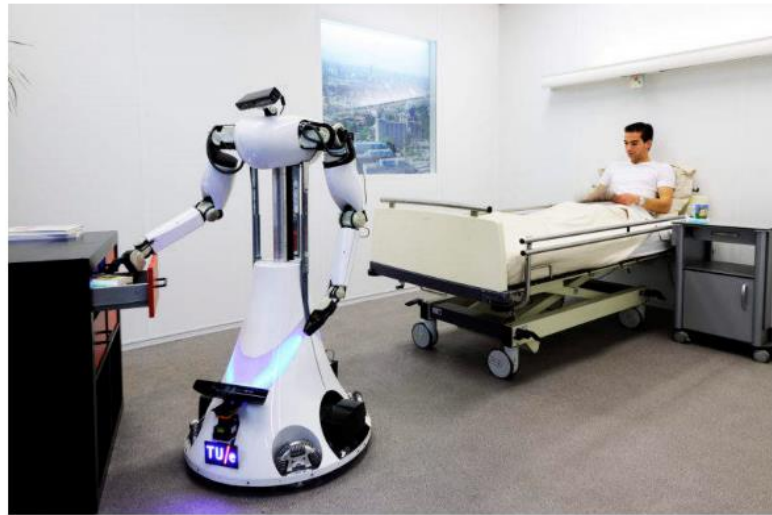

(d)

Figure 8: Robotics in Healthcare

\section{Defense and Public Safety Industries}

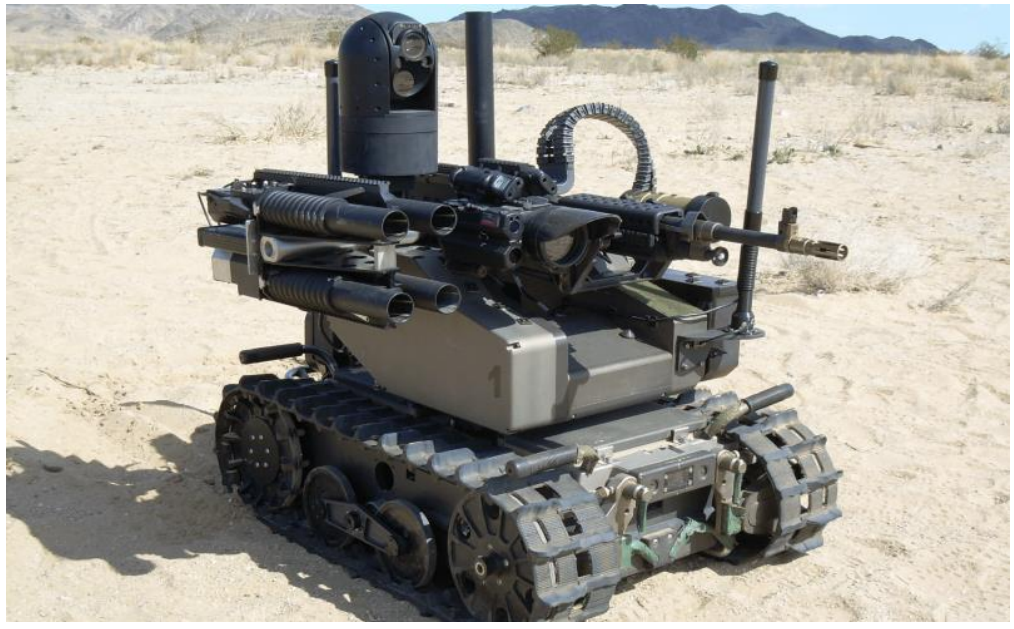

Figure 9: Defense Robot 
When people think about robotics that revolutionize an industry, they always first think of security or protection. The public has seen the military sector transform entirely because of the production of unscrewed vehicles and the robotics used to carry out identification, battlefield and centrifugal duties .. Drones were so successful in military applications that many firms like Amazon chose to use them for commercial purposes.

These types of robotics were also used by the public safety industry. Drones may be originally involved in auto crashes or other accidents. There are, for example, several companies which create remote-controlled, unscrewed flying drones that can evaluate and track potentially hazardous scenarios in real time. This kinds of drones are used for military and public security purposes. The way these two sectors track is also revolutionized by robotics.

\section{The MANUFACTURING INDUSTRY}

Global production began to use programmable factory robots in 1961. At the point, robots were automatic and performed repeated, menial tasks that people felt repetitive or dangerous. Established since then in the automotive sector, robots have now become much more effectively than unqualified labor.

For eg, Australia's Drake Trailers announced the incorporation into the production line of a single welding robot and a 60 percent improvement in efficiency, and also clever, often working and studying along with humans, to increase the amount of manufacturing tasks they can accomplish.

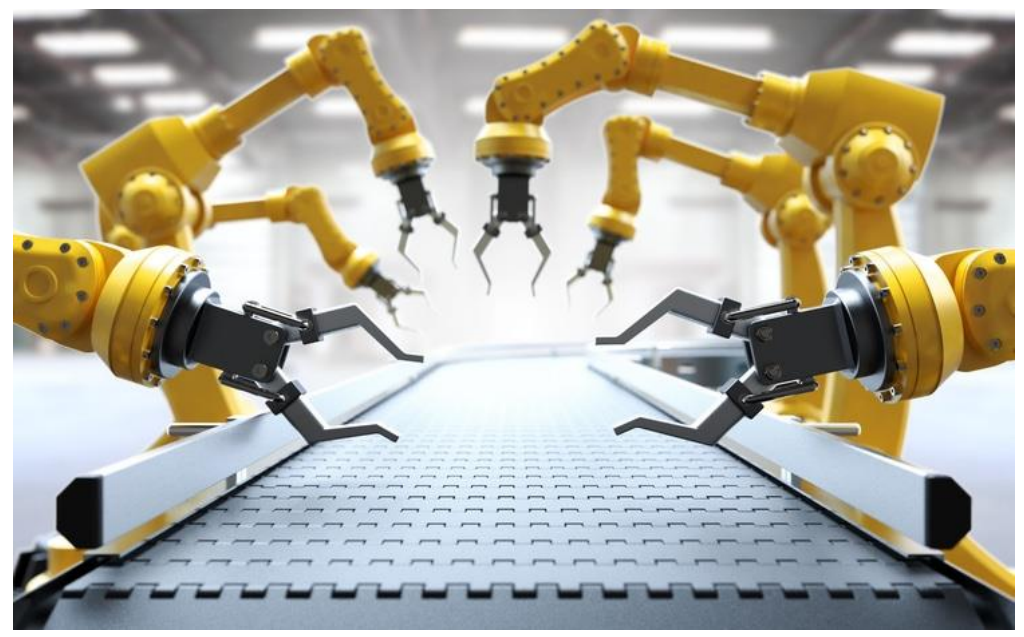

Figure 10: Manufacturing robot

\section{THE MinING INDUSTRY}

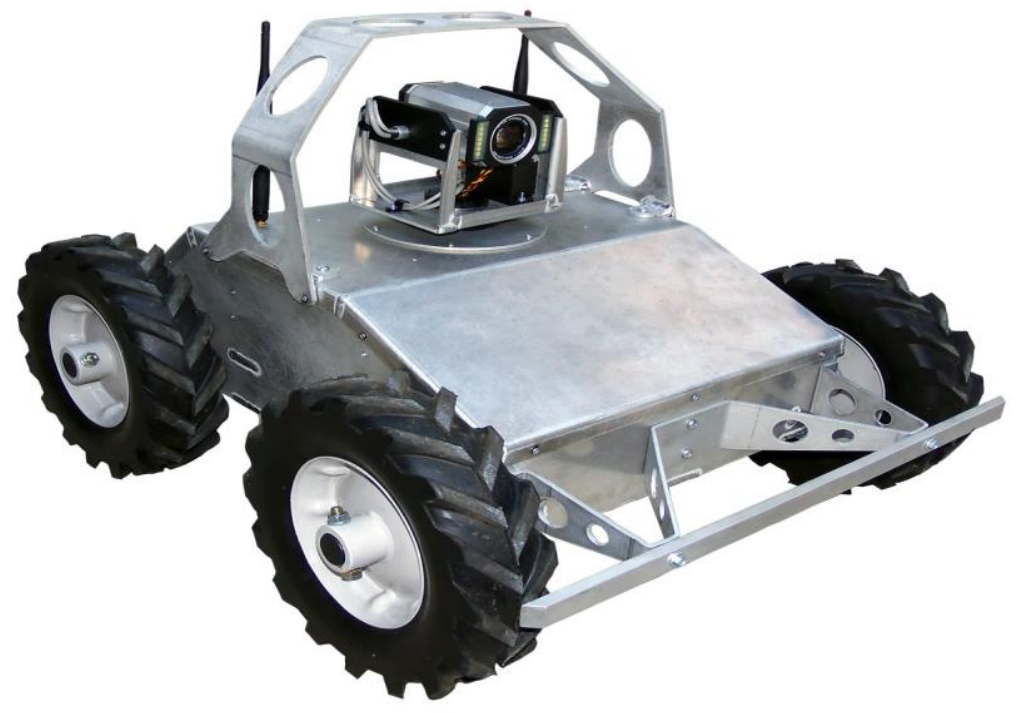

Figure 11: Mining robot 
Mining now depends mostly on automation and sophisticated robots, once relying on human resources. These robot types perform recognition and gather valuable inside knowledge. This provides the remaining human miners with a better work environment. Innovation from Stanley, for example, has an innovative, tailored robot which can be transported over dangerous terrain with a Segway mobility platform (RMP).

Additionally, the digging equipment itself has become extremely advanced in recent years. Currently, robotoperated drills can conduct drilling deep in the earth as well as offshore, allowing mining companies to dig deeper and in more treacherous conditions than if they had to rely on human operators.

\section{CONCLUSION}

In a profound learning method, we introduced a framework for detecting robots from RGBD results. Our process has many benefits over modern methods. In the first instance we can stop hand engineering functionality by using deep learning, instead of learning them. Secondly, our findings indicate that profound teaching methods transcend even well-designed hand-made features of previous work substantially. We have proposed a new multimodal data study algorithm based on group regularization. In detailed experiments we prove that this algorithm delivers better features than current profound learning methods for multimodal data for robotic grasp detection. Our tests and findings demonstrate that our two-stage profound learning system with group regularization can accurately detect grasp for a large variety of artifacts including those that the system has not previously seen. Many robotics problems need to use perceptual knowledge, but good engineering features can be complicated and time-consuming. We will expand our solution to a number of other problems in future work.

\section{REFERENCES}

Asadullah, A., Juhdi, N. B., Islam, M. N., Ahmed, A. A. A. and Abdullah, A. (2019). The Effect of Reinforcement and Punishment on Employee Performance. ABC Journal of Advanced Research, 8(2), pp. 47-58. https://doi.org/10.18034/abcjar.v8i2.87

Balasubramanian, R., Xu, L., Brook, P. D., Smith, J. R., and Matsuok, Y. (2010). Human-guided grasp measures improve grasp robustness on physical robot. 2010 IEEE International Conference on Robotics and Automation, Anchorage, AK, 2010, 2294-2301. https://doi.org/10.1109/ROBOT.2010.5509855

Ben-Ari, M., \& Mondada, F. (1970). Robots and Their Applications. Retrieved November 05, 2020, from https://link.springer.com/chapter/10.1007/978-3-319-62533-1_1

Bicchi A. (1995). On the Closure Properties of Robotic Grasping. The International Journal of Robotics Research, 14(4), 319-334. https://doi.org/10.1177\%2F027836499501400402

Bohg, J., Morales, A., Asfour, T. Kragic, D. (2014). Data-Driven Grasp Synthesis-A Survey. IEEE Transactions on Robotics, 30(2), 289-309. https://doi.org/10.1109/TRO.2013.2289018

Daniilidis, K., PF. Felzenszwalb, D., D. Fischinger, A., D. Guo, F., I. Lenz, H., AA. Maciejewski, C., . . X. Zeng, W. (1999). Robotic grasp detection based on image processing and random forest. Retrieved November 05, 2020, from https:/ /link.springer.com/article/10.1007/s11042-019-08302-9

Donepudi, P. K. (2017). Machine Learning and Artificial Intelligence in Banking. Engineering International, 5(2), 83-86. https://doi.org/10.18034/ei.v5i2.490

Donepudi, P. K. (2018). AI and Machine Learning in Retail Pharmacy: Systematic Review of Related Literature. $A B C$ Journal of Advanced Research, 7(2), 109-112. https://doi.org/10.18034/abcjar.v7i2.514

Donepudi, P. K. (2019). Automation and Machine Learning in Transforming the Financial Industry. Asian Business Review, 9(3), 129-138. https://doi.org/10.18034/abr.v9i3.494

Dragan, A. D., Lee, K. C., Srinivasa, S. S. (2013). Legibility and predictability of robot motion. 2013 8th ACM/IEEE International Conference on Human-Robot Interaction (HRI), Tokyo, 301-308. https://doi.org/10.1109/HRI.2013.6483603

Ioffe, S. \& Szegedy, C. (2015). Batch Normalization: Accelerating Deep Network Training by Reducing Internal Covariate Shift. Proceedings of the 32nd International Conference on Machine Learning, in PMLR 37:448-456. https://arxiv.org/abs/1502.03167

LeCun, Y., Bengio, Y. \& Hinton, G. (2015). Deep learning. Nature 521, 436-444. https:/ / doi.org/10.1038/nature14539 
Lenz, I., Lee, H., \& Saxena, A. (2015). Deep learning for detecting robotic grasps. The International Journal of Robotics Research, 34(4-5), 705-724. https://doi.org/10.1177/0278364914549607

Maleque, R., Rahman, F., \& Ahmed, A. A. A. (2010). Financial Disclosure in Corporate Annual Reports: A Survey of Selected Literature. Journal of the Institute of Bangladesh Studies, Vol. 33, 113-132. https://doi.org/10.5281/zenodo.4008320

Quach, K. (2019). Watch an AI robot program itself to, er, pick things up and push them around. Retrieved November 04, 2020, from https://www.theregister.com/2019/01/18/ai robot programs itself/

Saxena, A., Driemeyer, J., \& Ng, A. Y. (2008). Robotic Grasping of Novel Objects using Vision. The International Journal of Robotics Research, 27(2), 157-173. https://doi.org/10.1177/0278364907087172

Yamanobe, N., \& Nagata, K. (2010). Grasp planning for everyday objects based on primitive shape representation for parallel jaw grippers. 2010 IEEE International Conference on Robotics and Biomimetics, 1565-1570.

--0 -- 